\title{
Image type-based Assessment of SIFT and FAST Algorithms
}

\author{
Muthukrishnan, $\mathrm{R}^{1}$ and $\mathrm{Ravi}, \mathrm{J}^{2}$ \\ ${ }^{1}$ Assistant Professor Department of Statistics, Bharathiar University, Coimbatore, \\ India \\ ${ }^{2}$ Assistant Professor Department of Statistics, PGP College of Arts \&Science, \\ Namakkal, India \\ muthukrishnan70@rediffmail.com ${ }^{1}$,raviking2008@gmail.com
}

\begin{abstract}
Identifying the interest points in an image is a key step in image processing and computer vision tasks. Every corner of the images represents a lot of information. Extracting the true corners is the main object to image processing, which can reduce much of the time and calculations. Many algorithms have been suggested in the image processing to detect the true corners, based on the robust statistics. In this paper the corner detection algorithms SIFT and FAST have been studied in image processing under the various image formats. Also, it can provide a direction to the researchers to use the algorithm for the suitable image format and to develop a new algorithm which can detect the exact corners of an image/blurred image. The FAST corner detection method compared with the results of SIFT corner detection method. Experimental results show that the FAST corner detection gives better results compared to SIFT method. All the experiments are carried out MATLAB software.
\end{abstract}

Keywords: Corner detection, SIFT, FAST, robust features

\section{Introduction}

Corner detection is a vital research area in computer vision. Corner is an important local feature in images. Corner detection has played an important role in image matching [1], outline capturing system [2-3], image representation [4] and other fields. The gray level corner detection can be classified four performances of robustness and it must be specified all of the corner detection, such as detection, localization, stability and complexity. Corner and edges are the main role in image matching. The SIFT and FAST corner detection are the recently developed method for image matching.

The scale variation and rotations SIFT can give better performance and the recently focuses on FAST corner point detection through machine learning approach that has good and better performance and also low resource requirements. There are recently developed many applications that are related to corner detection, including image matching, image stitching, object identification and stereo matching, among many others.

A corner can be defined as the intersection of two edges. The result of image processing directly affected for true and quality of the corners. The corner detection, keeps useful information and improving the efficiency. A number of corner detectors have been proposed by the researchers. A variety of quantitatively evaluation methods of corner detection algorithm have been proposed [5-7].

FAST and SIFT method are characterized by its speed and its independence to other local features, using corners own features to detect corners directly. In this paper, these two corner 
detection algorithms were compared theoretically and their efficiency have been quantitatively analyzed with various types of image/blurred image by using MATLAB software. An elaborate discussion about SIFT and FAST algorithms are given in section 2. Section 3 presents the experimental results based on SIFT and FAST corner detection algorithm. The summary and discussion are made in the last section.

\section{Corner Detection Algorithms}

\section{A. SIFT Corner Detection Algorithm}

SIFT (Scale Invariant Feature Transform) corner detection is realized by extracting distinctive invariant features from images, which was proposed by DAVID G. LOWE (2004) [8]. The SIFT features are highly distinctive and correctly matched with high probability against a large database of features from images. This approach can robustly identify objects and achieving near real time performance. This method has four major stages and they are used to generate the set of image features such as Scale space extrema detection, keypoint localization, orientation assignment, and key point descriptor. This method transforms image into scale invariant coordinates relative to local features. The scale space of the image of the function,

$$
\mathrm{L}(\mathrm{x}, \mathrm{y}, \sigma)=\mathrm{G}(\mathrm{x}, \mathrm{y}, \sigma) * \mathrm{I}(\mathrm{x}, \mathrm{y})
$$

Where $\mathrm{L}(\mathrm{x}, \mathrm{y}, \sigma)$ is produced from the convolution of a variable and $\mathrm{G}(\mathrm{x}, \mathrm{y}, \sigma)$ is the scale Gussian with input image $\mathrm{I}(\mathrm{x}, \mathrm{y})$ and $*$ is the convolution operator in $\mathrm{x}$ and $\mathrm{y}$. David $\mathrm{G}$. Lowe (1999) proposed using scale space extrema in the different of Gussian function is

$$
\begin{aligned}
\mathrm{D}(\mathrm{x}, \mathrm{y}, \sigma) & =\left[(\mathrm{G}(\mathrm{x}, \mathrm{y}, \mathrm{k} \sigma)-\mathrm{G}(\mathrm{x}, \mathrm{y}, \sigma)]^{*} \mathrm{I}(\mathrm{x}, \mathrm{y})\right. \\
& =\mathrm{L}(\mathrm{x}, \mathrm{y}, \mathrm{k} \sigma)-\mathrm{L}(\mathrm{x}, \mathrm{y}, \sigma)
\end{aligned}
$$

The DOG function will have a strong response along edges, even if the location along the edge is poorly determined and therefore unstable to small amounts of noise. The principal curvatures can be computed from a $2 \times 2$ Hessian matrix at the location and scale of the key point,

$$
\mathrm{H}=\left[\begin{array}{ll}
D_{x x} & D_{x y} \\
D_{x y} & D_{y y}
\end{array}\right] \text { and reject those for which } \frac{\operatorname{tr} H^{2}}{\operatorname{det} H}>10
$$

The SIFT key points distinctiveness is achieved by assembling a high-dimensional vector representing the image gradients within a local region of the image. The key points have been shown to be invariant to image rotation and scale and robust across a substantial range of affine distortion, addition of noise, and change in illumination. Large numbers of key points can be extracted from typical images, which leads to robustness in extracting small objects among clutter. The SIFT key points perform well for images subject to noise/blur.

\section{B. FAST Corner Detection}

The several feature detectors, many of them, are really good. The FAST (Features from Accelerated Segment Test) algorithm was proposed by Edward Rosten and Tom Drummond [9]. This method has three advances, first we present a new heuristic for feature detection, second, we generalize the detector and finally, we carry out a rigorous comparison of corner 
detectors based on the above repeatability criteria and also this method has both very fast and very high quality [10]. For each location on the circle $\mathrm{x} \in\{1,2 \ldots, 16\}$, the pixel at the position relative to, denoted by $\mathrm{p} \rightarrow \mathrm{x}$, can have on the three states,

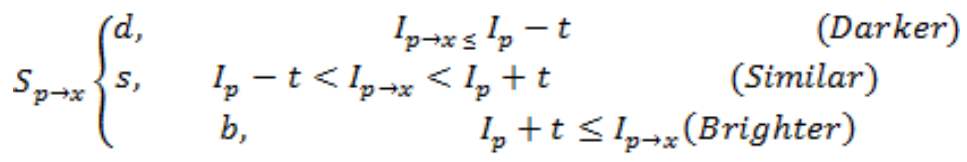

The total entropy of $\mathrm{k}$ for any arbitrary set of corners, $\mathrm{Q}$ is

$$
\mathrm{H}(\mathrm{Q})=(\mathrm{c}+\mathrm{c} *) \log _{2}(\mathrm{c}+\mathrm{c} *)-\operatorname{cog}_{2}-\mathrm{c}^{*} \operatorname{loc}_{2} \mathrm{c}^{*}
$$

Where $\mathrm{c}$ is the number of true corners and $\mathrm{c}^{*}$ is the number of false corners. The repeatability, $R$, is defined to be

$$
\mathrm{R}=\mathrm{N}_{\text {Repeated }} / \mathrm{N}_{\text {useful }}
$$

In an image, identify an interest point, then considering a circle of sixteen pixels around the pixel. Find the number of contiguous pixel to the interest point with respect to threshold value T. This version does not perform well when the number of contiguous pixels less than 12 . To improve the speed and efficiency the author has introduced the machine learning approach. Detection of multiple interest points adjacent to one another can be dealt with by applying non maximal suppression after detecting the interest points.

The speed and complexity of an algorithm must meet the demand of real-time task, that is the algorithm should be fast enough to be sable in the final image processing system. The runtime of an algorithm can describe its complexity [11]. The criterion of repeatability is first introduced to measure the consistency of key point location [12]. It is based on the number of points that appear repeatedly between two images by using the concept of holography. However the execution is too complex to be measured using the smart phone. After all, we need to know the exact view change to estimate the location of the key points.

\section{Experimental Results}

This section presents the relative performance of SIFT and FAST corner detection. The experiments are carried out using MATLAB, and tested with an image. To study the performance of the SIFT and FAST corner detection techniques, a number of experiments were carried out with a real image [(321x256), $(9.12 \mathrm{~KB}), \mathrm{JPG}]$ and it's of various types along with salt and pepper noise. This image has approximately 96 corners. The number of true/(false) corners detected under these algorithms and the time taken to detect the corners are summarized in the Table 3.1 and corners detected images are displayed in the Figure 3.1.

\begin{tabular}{|c|c|c|c|c|c|c|c|}
\hline \multirow{3}{*}{ Type } & \multirow{3}{*}{$\begin{array}{c}\text { Size } \\
\text { (in KB) }\end{array}$} & \multicolumn{6}{|c|}{ No. of corner detected } \\
\hline & & \multicolumn{3}{|c|}{ SIFT } & \multicolumn{3}{|c|}{ FAST } \\
\hline & & Time & True & False & Time & True & False \\
\hline JPG & $9.12(10.7)$ & $0.0212(0.1114)$ & $59(46)$ & $13(21)$ & $0.0191(0.0199)$ & $68(61)$ & $11(15)$ \\
\hline BMP & $241.00(82.5)$ & $0.0316(0.2845)$ & $51(41)$ & 14(30) & $0.0198(0.1365)$ & $61(52)$ & $12(21)$ \\
\hline
\end{tabular}

Table 3.1. Processing Time and Corners Detected under SIFT and FAST with Various Type of Images 


\begin{tabular}{|l|c|c|c|c|c|c|c|}
\hline GIF & $18.60(19.5)$ & $0.0262(0.2431)$ & $61(56)$ & $23(31)$ & $0.0232(0.2142)$ & $65(57)$ & $18(17)$ \\
\hline PNG & $24.00(61.9)$ & $0.0362(0.1234)$ & $62(58)$ & $14(26)$ & $0.0203(0.1134)$ & $63(51)$ & $09(19)$ \\
\hline TIFF & $8.59(74.9)$ & $0.0179(0.1113)$ & $61(52)$ & $24(38)$ & $0.0208(0.1024)$ & $66(55)$ & $20(27)$ \\
\hline
\end{tabular}

\begin{tabular}{|c|c|c|c|c|c|}
\hline Type & JPG & BMP & GIF & PNG & TIFF \\
\hline $\begin{array}{l}\text { Original Image } \\
\text { (without noise) }\end{array}$ & 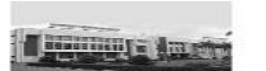 & 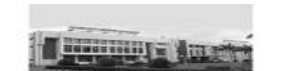 & 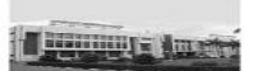 & 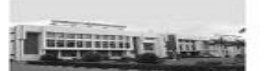 & 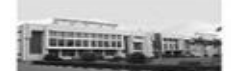 \\
\hline SIFT & 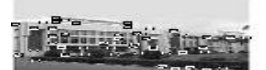 & D I & Iftabis boen & 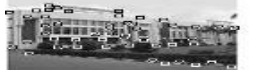 & 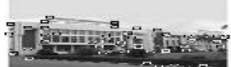 \\
\hline FAST & 1.0. & 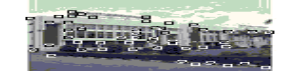 & 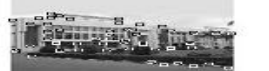 & Ni: & 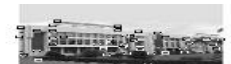 \\
\hline $\begin{array}{l}\text { Original Image } \\
\text { (with noise) }\end{array}$ & 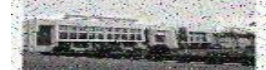 & 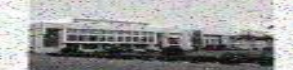 & 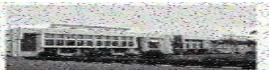 & 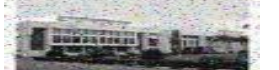 & 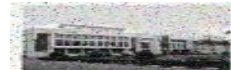 \\
\hline SIFT & 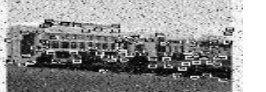 & Chertixy & & Simbint & 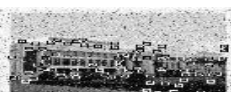 \\
\hline FAST & If & 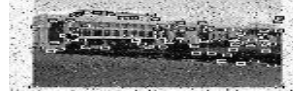 & 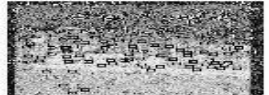 & 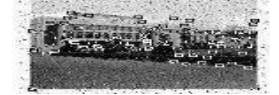 & 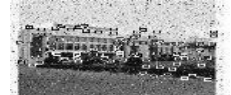 \\
\hline
\end{tabular}

Note: ( ) results based on salt and pepper noise image

Figure 3.1. Images Along with Corners Detected under SIFT and FAST

It is observed that the FAST algorithm performs well by considering the criterions, time true/false corners detected, type of images/blurred image. All the matching file formats of the image FAST corner detection gives best result when compared to SIFT. The more number of true corners are detected in the image which supports JPG and TIFF image file formats.

\section{Summary and Discussion}

Corner detection plays a vital role in performing computer vision tasks. A many of the corner detectors are exist in the literature, but still it is a challenging task of the researcher to find the efficient detector in the context of speed, number of true corners, type image file format, blurred/noise image etc., In this context this paper emphasizes the SIFT and FAST corner detectors and their performance has been studied, quantitatively evaluated and it is concluded that FAST algorithm provides better results when compared with SIFT by considering the specific criterions. SIFT algorithm consumes too much time in the computation thus results in lagging on the image that reduces the image quality significant. Only FAST achieves the real-time performance in the corner detection in an embedded device. However the FAST algorithm is not significantly high when compared to SIFT corner detection method while considering the feature illumination changes. It is observed that the development of these algorithms mainly based on the traditional statistical methods. But the 
traditional methods are very sensitive in the context of noise/outliers. The modern robust statistical methods, which were developed by statistical communities recent past can tolerate the presence of data points that do not obey the assumed model namely outliers/noise. The researcher should aware the existence of robust methods and develop the new algorithm to perform computer vision tasks in an efficient manner.

\section{References}

[1] T. Kim and Y. J. Im, "Automatic satellite image registration by combination of matching and random sample consensus", IEEE Trans on Geo science and Remote Sensing, vol. 41, no. 5, (2003), pp.1111-1117.

[2] M. Sarfaz, M. R. Asim and A. Masood, "Capturing outlines using cubic Bezier curves", Proceeding of IEEE $1^{\text {st }}$ Commination Technologies: From Theory to Application, (2004), pp. 539-540.

[3] Z. Wang, J. Chen and L. Dou, "Extracting Foot print Contour Rapidly by Curve Evolution via Level sets", Journal of Computer-Aided Design \& computer Graphics, vol.19, no. 10, (2007), pp. 1269-1273.

[4] C. A. Cebrelli and U. M. Molter, "Automatic representation of binary images", IEEE Tran on pattern Analysis and Machine Intelligence, vol. 12, (1990), pp. 1190-1196.

[5] Y. Yang and T. Zhang, "Assessing criterion of corner finders", Journal of Harbin Institute of Technology, vol. 30, no. 2, (1998), pp. 7-10.

[6] C. Schmid, R. Mohr and C. Bauchage, "Evaluation of interest point detectors", Internal Journal of Computer Vision, vol. 37, no. 2, (2000), pp. 51-172.

[7] F. Mokhtarian and F. Mohanna, "Performance of evaluation of corner detectors using consistency and accuracy measures", Computer vision and Image Understanding, vol. 102, no. 1, (2006), pp. 81-94.

[8] David G. Lowe, "Distinctive Image features from Scale Invariant Key points", Internal Journal of Computer Vision, vol.60, no. 2, (2004), pp. 91-110.

[9] E. Rosten, R. porter and T. Drummond, "Faster and better: a machine learning approach to corner detection", IEEE Trans. Pattern Analysis and Machine Intelligence, vol. 32, (2010), pp.105-119.

[10] E. Rosten and T. Drummond, "Fusing Points and Lines for High Performance Tracking", British Machine Vision Conference, (2003), pp. 719-728.

[11] J. Chen, L.-h. Zou, J. Zhang and L.-h. Dou, "The comparison and Application of Corner Detection Algorithms", Journal of Multimedia, vol. 4, no. 6, (2009), pp. 435-441.

[12] C. Schmid, R. Mohr, and C. Bauckhage, "Evaluation of Interest Point Detectors", International Journal of Computer Vision, vol. 37, no. 2, (2000), pp.151-172. 
International Journal of Signal Processing, Image Processing and Pattern Recognition Vol.8, No.3 (2015) 